\title{
Principles of Nature
}

\section{Apollinarii Stetsun}

Frantsevich Institute for the Problems of Materials Science, National Academy of Sciences of Ukraine,

Kyiv, Ukraine

Email: stetsun387@gmail.com

How to cite this paper: Stetsun, A. (2018) Principles of Nature. Journal of Modern Physics, 9, 320-334.

https://doi.org/10.4236/jmp.2018.93023

Received: December 27, 2017

Accepted: January 30, 2018

Published: February 2, 2018

Copyright (C) 2018 by author and Scientific Research Publishing Inc. This work is licensed under the Creative Commons Attribution International License (CC BY 4.0).

http://creativecommons.org/licenses/by/4.0/

\begin{abstract}
The analysis of peculiarities of structures of different physical systems, such as hydrogen atom, Solar system, a star with surrounding objects has shown that one of the important features of matter existence is that matter is defined by functions or parameters that strive for maximum or maximums. As a result a principle of matter, existence has been formulated which states that matter strives for maximum or maximums formation. Another principle that information strives toward maximum or maximums has been formulated on the basis of Darwin's theory and certain concepts of genetics. The theory that has been developed accounts for maximalization processes in nature. This theory does not neglect principles of minimization.
\end{abstract}

\section{Keywords}

Nature, Maximalization, Matter, Information

\section{Introduction}

What tendencies and principles of nature are the most globally important and essential? Nature is diverse and enormous encompassing a huge quantity of bodies and phenomena. The wide range of objects from the most distant galaxies of the Universe to the smallest pieces of sand on the Earth, as well as the tiniest elementary particles, all comprise nature in which we are used to distinguish between living and non-living things. Are there any most general regularities common for this huge array of objects in the material world of objects and phenomena? What common laws govern the giant stars in space and life on Earth? What features are common for the structure of the Solar system and the structure of atoms? The present work represents an attempt to answer these questions, to show that they can be answered from the standpoint of trends common to physics as a whole. 


\section{Theoretical Foundations of the Analysis}

In order to find the most important conformities of nature it is necessary to analyze its most general phenomena and constituent parts.

As physics developed it became evident that many physical phenomena as well as the composition of substance rely on the atomic structure [1] [2] [3] [4].

According to the ideas generally accepted by scientific community [3] [4] atom contains in its center a heavy positively charged nucleus surrounded by lighter negatively charged electrons moving around it. The state of atom can be determined by Shrodinger's equation. For the atom of hydrogen, the equation is solved exactly, so we shall consider this case in more detail.

Shrodinger's equation for the atom of hydrogen can be written as follows [4]:

$$
\frac{\hbar^{2}}{2 m_{0}} \Delta \psi+\left(W+\frac{e^{2}}{4 \pi \varepsilon_{0} r}\right) \psi=0
$$

where $e$ is the charge of electron, $m_{0}$ is the mass of electron, $r$ is the distance between the nucleus and electron, $W$ is the electron's energy. The equation has the following solution:

$$
\psi_{n l m}=R_{n l}(r) \Theta_{l}^{m}(\theta) \Phi_{m}(\varphi)
$$

where $n$ is the main quantum number, $l$ is the orbital quantum number, $m$ is the magnetic quantum number. $R_{n l}(r)$ is the radial wave function, $\Theta_{l}^{m}(\theta)$ and $\Phi_{m}(\varphi)$ are angular wave functions.

The squared module of the wave function $\left|\psi_{n l m}\right|^{2}$ determines the density of the probability to find the electron at the point with certain coordinates [4] [5] [6]. Instead of speaking about the density of probability it is common to use another term-the density of electron cloud. The electron belonging to hydrogen is brought into correspondence with an electron cloud. The distribution of electron cloud density around the atomic nucleus is completely determined by the quantity $\left|\psi_{n l m}\right|^{2}$. The distribution of electron cloud density along radius is given by the function $r^{2}\left|R_{n l}\right|^{2}$. Such distribution has been calculated [7] and the results are shown in Figure 1. The similar distribution is given in Ref. [4]. Main quantum number $n$ determines the energy of hydrogen atom. The values of quantum numbers $n$ and $l$ have influence on of the distribution of electron cloud density along radius. Orbital quantum number 1 may be equal from $1,2,3$, to $n-1$.

A deep insight into the atomic structure can be obtained by comparing the mass density of nucleus and mass density of electron

The classical estimation of electron radius is [2]:

$$
r_{0}=\frac{e^{2}}{m_{e} c^{2}}=2.81777 \times 10^{-13} \mathrm{~cm}
$$

Electron mass $m_{e}=9.109548 \times 10^{-28} \mathrm{~g}$, therefore the electron mass density is the following: 

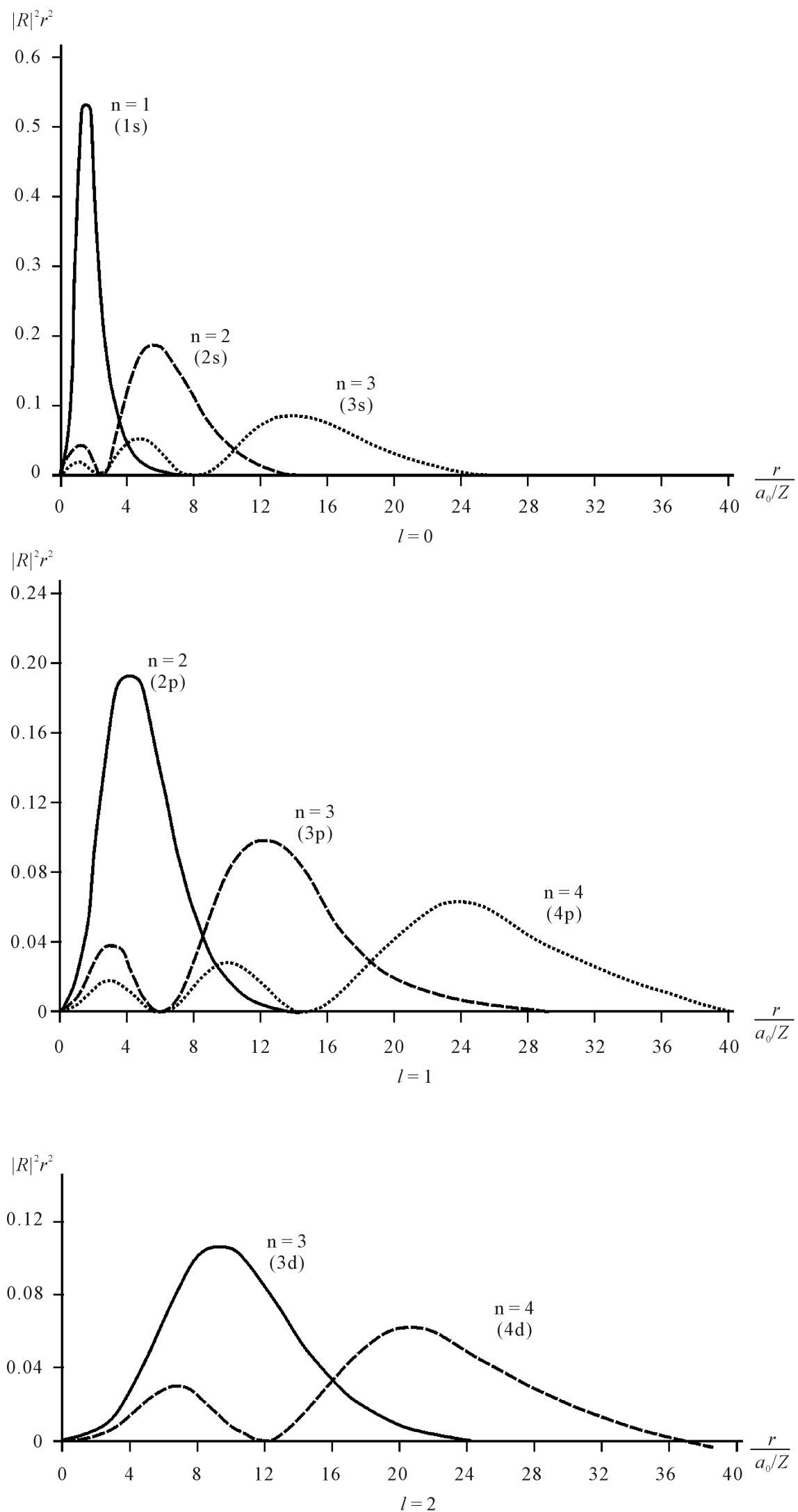

Figure 1. Radial dependence of probability density in quantum mechanics atom with one electron. $a_{0}$ is the radius of Bohr's orbit. 


$$
\rho_{e}=\frac{m_{e}}{\frac{4}{3} \pi r_{0}^{3}}=9.73 \times 10^{9} \frac{2}{\mathrm{~cm}^{3}}
$$

Since nucleus is a particle or, in general case, a system of particles obeying the laws of quantum mechanics, its size can be found only with an accuracy allowed by the ratio of indeterminate forms. The experiments on neutron scattering by atomic nuclei from which the sizes of nuclei were estimated have shown that nucleus sizes increase with the increase of the element atomic number by the law [1] [8]:

$$
R=R_{0} \sqrt[3]{A}
$$

where $R$ is the radius of nucleus, $R_{0}=1.4-1.5 \times 10^{-13} \mathrm{~cm}, A$ is the number of nucleons. Hence the value of mass density for hydrogen atomic nucleus can be calculated as follows:

$$
\rho_{N}=\frac{M}{\frac{4}{3} \pi R^{3}}=1.45 \times 10^{14} \frac{2}{\mathrm{~cm}^{3}}
$$

where the hydrogen nucleus mass $M=1.6726513 \times 10^{-24} \mathrm{~g}$.

Thus, the calculations show that the ratio of nucleus mass density to electron mass density in hydrogen atom is $1.49 \times 10^{4}$, i.e. the nucleus mass density of hydrogen atom is greater than the electron mass density by a factor of nearly 15,000. This result follows from Equations (3)-(6) and the values $\rho_{N}$ and $\rho_{e^{*}}$

If we try to express the essence of the above estimation outlining the principle features, we shall note that the nucleus represents an extreme maximum of density in the structure of hydrogen atom, which is the most important conclusion from the above analysis.

As atomic physics developed hydrogen atom began to be treated as the simplest model structure. The other atoms contain greater numbers of nucleons in a nucleus and electrons in the shells surrounding a nucleus [1] [2] [3] [4]. Such atomic structure allows us to claim that the ratio of nucleus mass density to electron mass density in the atoms should be close to the corresponding value for hydrogen atom. Therefore it can be supposed that the same basic principle holds in all the other atoms and the nucleus of an atom will always be an extreme maximum of mass density. This assumption is important for our further analysis.

Another conclusion that can be made from the above considerations is that the electron in hydrogen atom can be treated as the second, much weaker maximum. The distribution of radial density of electron cloud in hydrogen atom is shown in accordance with modern quantum mechanics ideas in Figure 1 for different quantum numbers. The figure shows well pronounced maximums in electron density distribution, therefore hydrogen atom can be treated as the main maximum associated with the atomic nucleus and the second, much weaker maximum (or maxima) in the mass density associated with electron/electrons. This interpretation is consistent with the fact that the nucleus 
mass exceeds the electron mass by a factor of nearly 1840. As known from [1] [2] [8], the atomic nucleus mass of hydrogen $M=1.6726513 \times 10^{-24} \mathrm{~g}$, while the electron mass $m_{e}=9.109548 \times 10^{-28} \mathrm{~g}$. Such distribution of nucleus and electron mass also indicates that hydrogen atom can be treated as the main and additional maxima but now not only of mass density but also of mass itself. Since electron is an elementary particle possessing certain mass and in terms of quantum mechanics models is located within the atom boundaries its probability density should have a maximum or maxima. An example of such distribution is shown in Figure 1.

The importance of the widely accepted views on an atom as that containing electron shells is seen from the Physics encyclopedia [1]: "In complex atoms electrons are grouped in the shells surrounding a nucleus at different distances and are characterized by a certain value of electron mass density." That is, every electron shell can be brought into correspondence with the maximum of probability density of wave function. Figure 2 illustrates the radial distribution of charge density on the example of $\mathrm{Rb}^{+}$ion calculated by Hartree method in [7]. The mean charge densities are shown by dashed lines and correspond to individual electrons-1s, $2 \mathrm{~s}, 2 \mathrm{p}, 3 \mathrm{p}$ and $3 \mathrm{~d}$. It is evident that the maxima of accumulation curve are due to the available group of electrons with the same principal quantum numbers, which supports the assumption about the distribution of electrons in discrete separate atomic energy layers levels or shells. Similar plots are given in [7] for other atoms of chemical elements. Thus, microcosm is characterized by the existence of maximums and it is natural to ask a question if this peculiarity is also characteristic of macrocosm.

It should be noted that atomic structure was first compared with planetary structure of the Solar system at the time when Rutherford and Bohr worked out the planetary model of atom [3] [4], in which the positions of electrons on the orbits around atomic nucleus in the center are similar to positions of planets on their orbits around the Sun. In this paper, we shall also consider the structure of

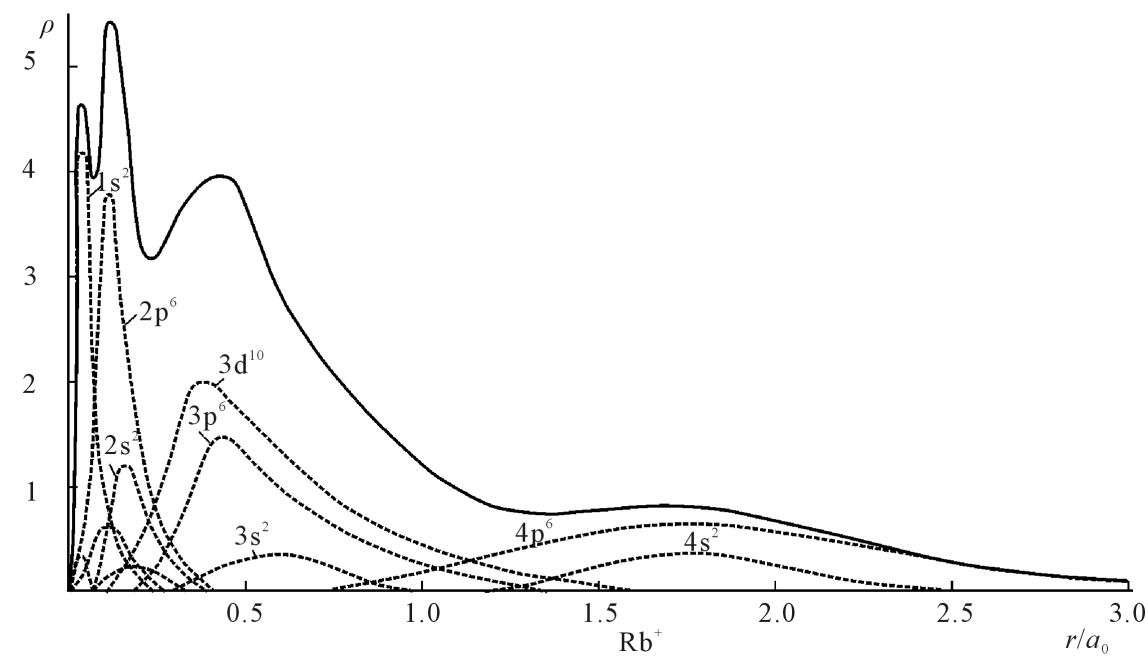

Figure 2. Radial distribution of charge density in $\mathrm{Rb}^{+}$ion. 
the Solar System, but for our purposes we shall analyze certain aspects in more detail.

One of the most important features of the Solar System is that the Sun is the central body in which $99.866 \%$ of the system's total mass is concentrated. Table 1 presents the data about the masses of Sun and the planets of the Solar System [2]. If we describe the mass distribution in the Solar System mathematically we shall obtain a pronounced maximum in the distribution of mass among the bodies of the Solar System which corresponds to the system's central body, i.e. the Sun. Therefore the Solar System is also characterized by the presence of central maximum. It should be noted that the nucleus mass of hydrogen atom is greater than electron mass by a factor of 1840 , i.e. there is analogy to the structure of the Solar System with respect to the central maximum as well. For other atoms this analogy also proves true, since the nucleus in them is even more massive in comparison with hydrogen atom.

The logic of these speculations can be applied for analyzing how matter in the Universe is distributed in the form of stars. The stars, as a rule, by their mass considerably exceed planets, asteroids, comets, meteorites and other celestial bodies inhabiting the Universe. Accordingly, star is main maximum and additional weaker mass maxima can be brought into correspondence with the planets surrounding a star.

Thus the process of maximalization of matter is characteristic for both micro- and macrocosm. Certain material systems such as atoms of chemical elements or stars together with the planets surrounding them can be brought into correspondence with the principal and additional maximums. Since matter is distributed in this manner throughout the Universe, maximilization represents an important phenomenon of nature.

Matter strives for maximum:

$$
\text { matter } \rightarrow \text { maximum (or maximums) }
$$

Table 1. Masses of sun and planets of the solar system [2].

\begin{tabular}{cc}
\hline Names of the solar system objects & Mass, gramme \\
\hline Sun & $1.991 \times 10^{33}$ \\
Mercury & $3.24 \times 10^{26}$ \\
Venus & $4.87 \times 10^{27}$ \\
Earth & $5.98 \times 10^{27}$ \\
Mars & $6.39 \times 10^{26}$ \\
Jupiter & $1.90 \times 10^{30}$ \\
Saturn & $5.68 \times 10^{29}$ \\
Uranus & $8.72 \times 10^{28}$ \\
Neptune & $1.03 \times 10^{29}$ \\
Pluto & $1.79 \times 10^{26}-1.18 \times 10^{27}$
\end{tabular}


in the sense that maximums of mass or mass density are formed in the material systems discussed above.

The most general regularities of nature cannot be discussed without considering the fields of physics that deal with the systems containing a greater number of constituent parts-thermodynamics and statistical physics. The fundamentals of these sections of physics are formulated in principia, i.e. by the laws of thermodynamics, and the second law of thermodynamics is of primary interest for the purposes of the given analysis. Such scholarly issue as Physics encyclopedia containing five volumes says the following [2]: "In modern thermodynamics the second law of thermodynamics is formulated in a unified and most generalized form as the law of increasing entropy. According to this law the change of entropy $\delta S$ in any closed system in any real process satisfies inequality $\delta S \geq 0$; equality sign being valid for reversible processes. In equilibrium state the entropy of closed system arrives at maximum..." Thus in case of closed thermodynamic system the second law of thermodynamics says about entropy maximalization. Therefore, we can state that in this case entropy shows a tendency to arrive at maximum:

$$
\text { entropy } \rightarrow \max
$$

Moreover, in the case with entropy we have to deal with one more process of maximalization. Since the process is associated with one of the laws of thermodynamics acquires global importance. We regard this consideration as another proof of such analysis appropriateness in the study of maximalization in nature.

The next large class of natural objects is the world of living beings. Traditionally it is studied by biology. However, and this will be shown further on, here again the same physical principle works, which proves that the concept of maximalization is true for the whole nature. In order to prove this we shall turn to evolutionary and informational aspects of living nature.

The origin of species and evolution of life on the Earth was described in the known work by Charles Darwin [9]. However, this theory does not consider information-specific aspects of evolutionary process.

Throughout the whole book Darwin persuasively provided the evidences supporting the idea that species were not created independently of each other but originated from species in the way similar to variations [9]. The theory also explains perfect organization and co-adaptation of living beings inhabiting our planet, which was an important problem approached in the book.

In order to give scientific explanation to origin and evolution of species Darwin introduced the concept of natural selection and struggle for existence. It has been shown that the struggle for survival is inevitable result of the large rate with which all the organic creatures tend to increase their population [9]. The living creatures capable of reproducing must be destructed at a certain moment of their life, in certain season or at certain age, because the population would grow exponentially and become so huge that no habitat could be large enough to hold all the descendants (Malthus law). Since the number of off-springs is more than 
can be supported they have to struggle for survival. Darwin distinguished three most important form of this competition:

1) intraspecific competition, i.e. the struggle between individuals in the same species;

2) interspecific competition, i.e. the struggle for existence between individuals of different species;

3) the struggle of living being with the physical conditions of environment.

As a result the principle of natural selection advances and directs the evolution. The motive forces according to Darwin's theory are hereditary variation and natural selection. Hereditary variation is the variation of individual traits at the moment of living organism origination. The struggle for survival usually results in extinction of a great number of individuals in every generation of any species and to selective reproduction of individuals. The inevitable result of hereditary variation and severe competition is natural selection-predominant survival and reproduction of the most adapted individuals in every species. Natural selection leads to speciation due to preserved adaptations [9] [10]. Thus, the main inference of Darwinism is that in the process of evolution the best adapted and biologically strongest individuals survive.

Darwin's theory has played great role in explaining the process of evolution and remains important for present day science. However, classical Darwinism does not answer the question about the mechanism of heredity because the laws governing heredity are for the most part unknown [9]. The recent development of genetics has revealed hereditary mechanism which further was described in more details by molecular biology. The most important advance made by genetics was that it has found the structure in an organism that carries information governing heredity. These structures are genes, the stretches of DNA (or in some cases RNA). Thus, a material carrier of information, or micromechanism responsible for heredity has been found, which explains the way the hereditary information is transmitted.

One more important achievement of genetics was that it has proved that genes can easily mutate. Mutations are known to be natural or intentional inheritable changes of genetic material resulting in changes of various traits [10] [11].

It is also of wide knowledge that mutational variability together with combinational variability provide material for natural selection which leads to development of new species in the process of evolution [10] [11].

The comparison of Darwin's theory and genetics findings reveals the following tendency: due to physical, chemical or biological impact on information carriers in the biological organisms, i.e. on genetic material, the genetic material undergoes changes and a certain array of data is created. It may be nuclear or non-nuclear changes. Genetic variation appears as genetic recombination and mutation.

In turn, among the whole population of living organisms the biologically strongest individuals survive in the result of natural selection. It is these best 
adapted individuals that carry the most valuable and important information. Thus, from all the biological array of information every new generation acquires the most, or in other words, maximally valuable information. This means that in the process of natural selection the information strives toward the maximum:

$$
\text { information } \rightarrow \text { maximum (or maximums) }
$$

The information strives toward the maximum (or maximums) depending on what biological objects we consider-a family, a population or a group of different individuals. This tendency of information to approach toward maximum determines the way in which information develops in the world of living nature. The law stating that information tends to approach maximum is one of the most important laws of life.

The above considerations show that the world of living nature is governed by a certain physical principle. Although the living objects obey the laws of biology, the action of the physical principle, information strives toward the maximum, is also observed.

To sum up, the three basic principles are peculiar to both living and non-living nature:

matter strives toward maximum or maximums

matter aspires to maximum or maximums

matter $\rightarrow$ maximum (or maximums)

entropy strives toward maximum

entropy $\rightarrow$ maximum

information strives toward maximum or maximums

information aspires to maximum or maximums

information $\rightarrow$ maximum (maximums)

Moreover, it should be noted that to some extent the maximilazition process is also characteristic for light, i.e. electromagnetic field. Since light can be considered not only as a wave but also as quanta, every quantum can be put into correspondence with a maximum of energy.

Thus, the maximalization processes play important unifying role in nature. They are a manifestation of nature's unity. The essence of this unity is that for different natural systems there exists a unique characteristic function that strives toward the maximum and this "aspiration" determines the essential features of a natural process.

In view of the maximalization property of nature one may ask a logical question if maximalization process can be used in practice. If maximums of mass or mass density are favourable processes in the Universe, then certain beneficial processes should occur when mass maximums approach each other. It can be expected that mass maximums will either become greater or interact with each other. The simplest way to ensure such interaction is to make atoms come closer. Since atoms consist of the main and additional maximums of masses, which are an atomic nucleus and electrons around it, one can expect these maximums to 
interact. Actually, as known from chemistry, interactions of this kind take place in a multitude of cases. For instance, if hydrogen and oxygen atoms converge by mixing appropriate gases, water is formed. When atoms of hydrogen and chlorine approach each other in a mixture of these substances, a new substance which is hydrogen chloride is produced. There are many more examples of new substance formation due to convergence of atoms comprising the reacting components. Furthermore, the atoms of the same chemical element, when converging, also can interact with each other. For example, the interaction of two hydrogen atoms yields hydrogen molecule $\mathrm{H}_{2}$, the convergence of two oxygen atoms yields oxygen molecule $\mathrm{O}_{2}$, and so on. It is known from physics and chemistry that interactions between the electrons and nuclei are the processes described in terms of quantum mechanics, i.e. the above examples are interactions of the main and additional mass maximums which are nuclei and electrons respectively.

These processes indicate that maximalization is inherent to nature, this is a process which is intrinsic and beneficial to nature. Atoms can be considered as the main and considerably weaker maximums of mass or mass density. The existence of these maximums is inherent to nature and beneficial. However even greater maximalization is possible by creating the conditions of closing these maximums, i.e. atoms of the same substance or different substances, in the result of which new substances or molecules will be created.

The very existence of physical bodies in the macrocosm is the manifestation of maximalization process. A solid body is formed by means of quantum mechanical interaction of electrons and nuclei of different atoms, i.e. the interaction of maximums in microcosm resulting in formation of a maximum in macrocosm. The solid body formed in this way can be considered as a local maximum of mass or mass density with respect to the molecules of surrounding air if the body is at a distance from the other solid bodies. For example, if such body as a stone is formed, a huge number of atoms of the same or different substances are combined in one body. Their combination is a maximum of mass of certain magnitude in the total distribution of mass in a physical system such as planet, for example. In the given case it does no matter how large the maximum is by its absolute value, the main thing is that this maximum exists in principle. The definite size of the body and its mass evidences that nature needs exactly this specific object. Therefore, as soon as the body has been formed, the maximalization process interrupts at the intermediate stage.

By forming new substances from atoms, molecules from atoms, macrobodies from atoms the nature shows the tendency to continue and repeat maximalization. In other words, if a natural system such as substance consisting of atoms has already undergone maximalization, which is the existence of an atom itself, this does not mean that the process has terminated ultimately. If a great number of atoms assemble into a massive body, this is a reiteration and continuation of maximalization process. When entropy strives toward maximum, or informa- 
tion strives toward maximum, we deal with the processes of repeated maximalization of certain functions, but in this case these functions differ from those maximized at the previous stage. The only fact that a substance consists of atoms already indicates that mass maximums in the form of nucleus and electrons are available in atom. In other words, the existence of atoms is the first stage of maximalization. However, if the entropy of a closed thermodynamic system consisting of atoms of a certain substance strives, upon reaching the equilibrium, toward the maximum, this is the second stage of maximalization. The same is true for living nature in case when the information in biological processes tends to reach the maximum. Living organisms also are comprised of atoms, atoms have a nucleus and electrons-and this is the first stage of maximalization. The second stage of maximalization is that another function-information, which is different from mass, strives toward maximum.

Nature evolves due to maximalization. The existence of nuclei and electrons in atoms, the atomic character itself makes the substance heterogeneous. This is the very first precondition of natural evolution. If nature were completely homogeneous, the evolution would be impossible. But since nature is not homogeneous, the evolution proceeds in forming more complicated structures, such as molecules, non-living bodies and living creatures, from atoms. Maximalization of entropy in closed thermodynamic systems and maximalization of information for living organisms ensures further evolution of nature. Our analysis reveals the main principles of natural evolution: after a certain function (mass or mass density) reaches maximum at a certain stage, another function, which is characteristic for another natural process (entropy or information), strives to reach the maximum. However, nature may evolve in other ways, when different characteristic functions are striving to maximums simultaneously.

In non-living nature, as discussed earlier, maximalization is manifested in different ways. Gravitation, for example, as well as other phenomena, supports the idea that maximalization is inherent to nature. Gravitation is the attraction of bodies of a certain mass to each other and, if this is possible in certain physical conditions, these bodies strive to connect to each other. Since every physical body is a kind of a maximum, the tendency of the maximums to merge results in formation of a larger maximum, which proves the tendency of nature to maximalization.

Considering different processes from the point of view of maximalization, it should be noted that even though these processes are global they can have certain peculiarities. A vivid example of this is the existence of stars of different types. As mentioned above, a star in general case can be considered as a cosmic formation which is a maximum of mass or mass density in the outer space. It is known [12], however, that besides singular stars there are also binary or multiple stars. This fact proves that the natural processes of maximalization may result in formation of several, instead of one, maximums. Nevertheless, maximalization of matter in the form of stars is peculiar to nature, since, according to [12], 92\% 
of matter in space is concentrated in stars, and only $8 \%$ of matter is in the interstellar space. In fact, the amount of matter in the form of stars is more than by an order of magnitude greater than the amount of matter in the interstellar space.

The formation of several or multiple maximums is for matter as important a process as the formation of only one maximum.

From the above analysis the following two physical laws that determine maximalization processes in nature can be formulated:

1) Nature evolves in such a way that the characteristic functions describing certain natural processes strive to maximum (maximums) or minimum (minimums): maximum (maximums) of masses, maximum (maximums) of matter densities, or maximum (maximums) of probability densities of electron and nuclei distributions in atoms are formed and maximalization of other characteristic functions takes place as well-information for objects of living nature and entropy for closed thermodynamic systems.

2) In a number of physical situations the essence of the phenomena is the interaction of objects which are maximums of characteristic function with respect to the environment (for instance, masses or the quantity of atoms in an object), or which form the maximum of characteristic function (for instance, mass density for a solid body or maximums of probability density for electrons and nuclei in atoms). An example of these interactions is the gravitational interaction of stars and planets surrounding them, or the gravitational interaction between the bodies on Earth and the planet itself.

The first law reflects the importance of maximalization (and minimalization, i.e. in general case the formation of extremum) in nature. The second law shows that in addition to maximalization process as such, there occurs a process of interaction between material maximums.

The formulation of the first law also reflects the importance of minimalization of characteristic functions inherent to nature, i.e. the importance of all processes leading to extremums.

\section{Practical Aspects of the Principle Which States the Matter Strives for Maximum or Maximums Formation}

In the previous paragraph we have shown that the principle that matter strives toward maximum (or maximums) is sufficiently universal because it is valid for the objects of both macro and microcosm.

The very formulation of the principle "matter strives for maximum (or maximums)" suggests the possibility of mathematical expression of this principle. In the mathematical analysis there is a well known problem of obtaining the extremum of a function, i.e. a function maximum or minimum. Applying appropriate methods of mathematical analysis in the description of the principle that matter strives toward maximum (or maximums), one can calculate the states of different objects in both macro and microcosm. It is quite obvious that the 
theory of mathematical analysis should be supplemented by the theory of the branch of physics related to every specific kind of problems. For instance, to calculate the state of atoms by maximalization method it is necessary to involve the quantum mechanics theory, and to calculate the state of matter in a star it is necessary to involve the theory used by astrophysics.

The most general principles of search for the states of objects in macro and microcosm according to maximalization theory is maximalization (i.e. search for maximum or maximums) of the functions of density, mass, number of particles, or squared module of wave function depending on the problem to be solved.

For the function with several variables, which is most often used in physical problems, the necessary condition of function extremum is that the function differential should equal zero [13] [14] [15]. The differentiated function $f(P)=$ $f\left(X_{1}, \cdots, X_{n}\right)$ can reach the extremum only at a stationary point $P_{0}$, i.e. such a point that

$$
d f\left(P_{0}\right)=0
$$

Consequently, the extremal point of function $f(P)$ satisfies the set of equations

$$
f_{x_{i}}^{\prime}\left(x_{1}, \cdots, x_{n}\right)=0 \quad(i=1, \cdots, n)
$$

The sufficient condition of the extremum is that function $f(P)$ at point $P_{0}$ has:

1) maximum if $d f\left(P_{0}\right)=0, d^{2} f\left(P_{0}\right) \prec 0$ at $\sum_{i=1}^{n}\left|d x_{i}\right| \neq 0$ and

2) minimum if $d f\left(P_{0}\right)=0, d^{2} f\left(P_{0}\right) \succ 0$ at $\sum_{i=1}^{n}\left|d x_{i}\right| \neq 0$.

Therefore, applying mathematical calculus in searching for the maximum of function to solve a specific physical problem it is possible to obtain a new set of equations and to solve it for every such problem. One of the practical aspects of maximalization theory is the possibility to derive corresponding equations and to solve them.

However, in the most general case one can use not only the equations which are derived basing on the condition that the function differential equals zero if a necessary condition is satisfied. The principle that matter strives toward maximum (or maximums), information strives toward maximum (or maximums) as well as other maximalization principles provides a possibility to derive and calculate the most different equations. It should be noted that this approach is in agreement with Shrodinger equation or the equations derived from Shrodinger equation. Moreover, for this purpose the principles of variational calculus and mathematics of functionals can also be applied [1] [2] [6].

For example, in variational calculus there is a necessary condition of function extremum known as Legendre necessary condition [16]. For curve $y(x)$ to yield maximum for functional

$$
J=\int_{x_{1}}^{x_{2}} F\left(x, y, y^{\prime}\right) \mathrm{d} x \quad y\left(x_{1}\right)=y_{1} \quad y\left(x_{2}\right)=y_{2}
$$

it is necessary that at all points on curve $y(x)$ the second derivative of subintegral 
function by $y^{\prime}$ should be non-positive

$$
F_{y^{\prime} y^{\prime}}\left(x, y(x), y^{\prime}(x)\right) \leq 0 \quad x_{1} \leq x_{2} \leq x_{3}
$$

In variational calculus there are several conditions at which functional extremums are possible, but the aim of this work does not involve the description of all the conditions of the functional extremums.

The general principles of maximalization theory described in this paper were first suggested and reported in references [17] [18] [19].

The next important aspect of practical application of maximalization theory stems from the non-uniform dependence of the function describing a physical process on the function variables. If the function has maximum or several maximums, this does indicate that its value is essentially heterogeneous in a certain range of variable values. To consider the consequences of this functional peculiarity we shall keep in mind that quantum mechanics knows the problems whose solution is expressed in the form of wave functions with harmonic behavior. The example can be the passage of the particle through a potential barrier (tunneling). Due to the fact that wave function displays periodic and harmonic alteration of maximums and minimums the particle described by the function of this kind shows the property of passing through the barrier, although it is impossible to overcome it according to the laws of classical mechanics. It is known that the wave properties of particles become apparent during alpha-decay of atomic nuclei, auto emission of electrons in metals as well as in some other phenomena. Heterogeneous functions are more common than harmonic wave functions, which extends the possibilities provided by the function harmonicity.

While the wave function describes the property to penetrate through the potential barrier due to regular alteration of maximums and minimums, the heterogeneous function shows that the same effect can be due to irregular alteration of maximums and minimums. The main thing is that in both cases this becomes possible because of existence of maximums and minimums as such. This means that another practical application of maximalization theory is the possibility to extend harmonic wave functions to the case of heterogeneous functions to solve certain physical problems.

\section{Conclusions}

The main goal of the present work is to demonstrate the importance of maximalization processes in nature. Even if only one maximalization process occurred in nature, for example, maximalization of mass or mass density, this only fact would have a great importance. In nature, however, such processes as maximalization of entropy and information are observed, which is the manifestation of nature's unity. These principles play important roles in natural evolution.

The above maximalization processes observed in nature allow us to make the following generalizations:

1) matter strives for maximum or maximums of characteristic physical quan- 
tities:

matter $\rightarrow$ maximum or maximums

matter strive for maximum or maximums formation

2) information strives for maximum or maximums depending the biological object under consideration such as a species, a single individual, a group of individuals:

information $\rightarrow$ maximum or maximums

information aspires to maximum or maximums

3) entropy strives toward maximum in a closed thermodynamic system: entropy $\rightarrow$ maximum

The principles suggested in this work are treated as one of the important aspects of nature.

\section{References}

[1] Physical Encyclopedic Dictionary (1984) Enciclopediya, Moscow.

[2] Physical Encyclopedic Dictionary (1960) Enciclopediya, Moscow.

[3] Shpolskij, E.V. (1974) Atomic Physics. Nauka, Moscow.

[4] Belyj, M.U. and Ohrimenko, B.A. (1984) Atomic Physics. Visshaya Shcola, Kiev.

[5] Blohintsev, D.I. (1976) Quantum Mechanics. Nauka, Moscow.

[6] Fedorchenko, A.M. (1979) Quantum Mechanics. Visshaya shcola, Kiev.

[7] Freesh, S.E. (1963) Optical Spectra of Atoms. Fizmatgiz, Moscow.

[8] Lobodeuk, V.A, Ryaboshapka, K.P. and Shulishova O.I. (1975) Handbook of Physics. Naukova dumka, Kiev.

[9] Darwin, C. (1991) The Origin of Species. Nauka, St. Petersburg.

[10] Biology Encyclopedic Dictionary (1986) Enciclopediya, Moscow.

[11] Dubinin, N.D. (1979) General Genetics. Nauka, Moscow.

[12] Martinov, D.Y. (1979) The Course of General Astrophysics. Visshaya shcola, Moscow.

[13] Kudryavtsev, L.D. (1970) Mathematics Analysis. Visshaya Shcola, Moscow.

[14] Demidovich, B.P. (1977) The Collection of Tasks and Exercises in Mathematics Analysis. Nauka, Moscow.

[15] Korn, G. and Korn, T. (1978) Handbook in Mathematics. Nauka, Moscow.

[16] Mathematics Encyclopaedia (1982) Enciclopediya, Moscow.

[17] Stetsun, A.I. (2007) Author Rights and Adjacent Rights, 13, 87.

[18] Stetsun, A.I. (2009) Author Rights and Adjacent Rights, 18, 397.

[19] Stetsun, A.I. (2010) Author Rights and Adjacent Rights, 22, 246. 